\title{
TENDENCIAS E INCIDENCIA DE LOS FENÓMENOS MACROCLIMÁTICOS EN LA HIDROLOGÍA DEL ALTO Y MEDIO MAGDALENA
}

\section{TENDENCIES AND INCIDENCE OF MACROCLIMATIC PHENOMENA IN THE HYDROLOGY OF THE HIGH AND MIDDLE MAGDALENA BASINS}

Fecha de Recepción: 24 de septiembre de 2006 Aurora Velasco Rivera ${ }^{l}$ Mario Díaz Granados Ortiz ${ }^{2}$

Fecha de Aprobación: 30 de Octubre de 2006

RESUMEN: El objetivo de esta investigación se centra en llevar a cabo un análisis a lo largo del Río Magdalena en su cuenca alta y media para determinar qué estaciones muestran cambios estadísticamente significativos del caudal y cuál es la tendencia de estos cambios, con el fin de establecer pronósticos de la disponibilidad hídrica. Así mismo y para comprobar la incidencia de fenómenos macroclimáticos, se evalúa la correlación entre las series correspondientes al Indice Multivariado ENSO (MEI) y al índice de Oscilación del Atlántico Norte con las series de caudales en cada estación. Los resultados obtenidos confirman una correlación negativa con valores entre -0.15 y -0.505 para el índice MEI y una escasa correlación con el índice NAO. Se estima a largo plazo disminución de caudal en la zona.

ABSTRACT: The objective of this paper is to carry out an analysis along the Alto and Medio Magdalena River to determine changes and tendencies of flows statistically sig-nificant and water yield forecasts. In adition, a first approximation of correlation analysis among streamflows and Multivariate Enso Index (MEI) and North Atlantic Oscillation In-dex (NAO) is done in order to verify the incidence of macroclimatic phenomena in streamflow time series tendencies. The results obtained so far confirm a negative corre-lation with the values from -0.15 to 0.505 for the MEI index, and a slight correlation with the NAO index. It is expected, in the long term, a streamflow decrease in the region.

1 Docente Universidad Militar Nueva Granada, Programa de Ingeniería Civil, Magíster Ingeniería Civil

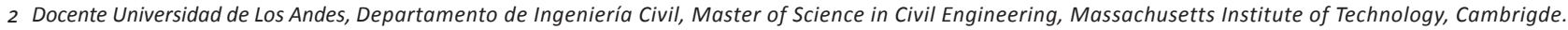
Magister en Ingeniería Civil 


\section{INTRODUCCIÓN}

La preocupación por la variabilidad climática y su influencia en las alteraciones de la precipitación, temperaturas y disponibilidad hídrica ha cobrado especial relevancia ya que éstas repercuten en las condiciones socioeconómicas de todos los habitantes del planeta. Entender tal variabilidad es muy importante para prevenir y mitigar los efectos adversos y para obtener los mayores beneficios de su correcta predicción. En la actualidad prestigiosas entidades a nivel mundial tales como el Pánel Intergubernamental de Expertos sobre el Cambio Climático (IPCC), la UNESCO, la Organización climática mundial (WMO), el Banco Interamericano de Desarrollo (BID), el CRID (Centro Regional sobre Desastres América Latina y EI Caribe) y los países afectados estudian la incidencia de los fenómenos macroclimáticos y de cambio climático sobre la disponibilidad hídrica, en búsqueda de modelos que pronostiquen la vulnerabilidad a los eventos extremos tales como sequías severas e inundaciones, entre otros.

En Colombia se han realizado varias investigaciones con el objeto de determinar la influencia de los fenómenos macroclimáticos en las alteraciones del clima a nivel nacional y regional. EI IDEAM, Instituto de Hidrología, Meteorología y Estudios Ambientales a través de su informe sobre los efectos naturales y socioeconómicos del fenómeno del Niño en Colombia, publicado en marzo de 2002 expone el avance del conocimiento acerca de la naturaleza y características principales del fenómeno El Niño, la descripción del fenómeno más fuerte, durante el periodo 1997-1998 y las recomendaciones para la reducción de impactos. Igualmente IDEAM, 2002 presenta en su informe No. 92 titulado Condiciones e Indicadores ambientales en Colombia, el estado de las condiciones climáticas e hidrológicas durante el desarrollo de El Niño 2002 con proyecciones a corto, mediano y largo plazo.
Otras entidades gubernamentales y universidades colombianas han hecho su aporte al estado del arte acerca del impacto de fenómenos macroclimáticos y de calentamiento global en la variabilidad climática y disponibilidad hídrica regional, como la Universidad Nacional, la Univer sidad de Los Andes y la Universidad del Valle.

Se ha utilizado una amplia documentación sobre el estado del arte, que sirvió de base para el presente trabajo así como información sobre la caracterización de la región del Valle del Alto y Medio Magdalena, registros de los datos de caudales mensuales del área y los datos sobre variaciones de índices macroclimáticos.

Para llegar a la meta propuesta todos los antecedentes anteriores se analizan, clasifican, sistematizan y redactan, y los datos correspondientes a caudales mensuales se someten a un proceso de análisis y tratamientos estadísticos con el fin de comparar y extraer conclusiones acerca de la influencia de estas variables en la hidrología de la región.

En el presente informe se presenta una reseña acerca del impacto de los fenómenos macroclimáticos en la hidrología nacional, una caracterización del valle Alto y Medio del Magdalena, una descripción de la metodología empleada en el análisis de las series de tiempo hidrológicas y los correspondientes resultados.

\section{MARCO CONCEPTUAL}

\section{A. Fenómeno El Niño}

El fenómeno El Niño-Oscilación del Sur (ENSO) es un evento natural que se da como resultado de la interacción entre el océano y la atmósfera en la región del Océano Pacífico Ecuatorial. El fenómeno tiene dos componentes, la componente oceánica que es 
El Niño y la componente atmosférica conocida como Oscilación del Sur (Poveda, 2005).

El Niño se desarrolla a través del calentamiento anómalo de las aguas superficiales del centro y el este del Océano Pacífico, el cual produce la profundización de la termoclina oceánica, el debilitamiento de los vientos alisios del este y el desplazamiento del centro de convección del oeste al centro del océano pacífico tropical.

La Oscilación del Sur (OS) es una onda estacionaria en la masa atmosférica que produce un gradiente de presiones entre el oeste y el este del Pacífico ecuatorial. Un centro de alta presión se localiza cerca de Tahití $\left(18^{\circ} \mathrm{S}, 150^{\circ} \mathrm{O}\right)$, mientras que un centro de baja presión se localiza en Indonesia y el norte de Australia cerca de Darwin $\left(12^{\circ} \mathrm{S}, 131 \mathrm{E}\right)$. Este gradiente de presiones constituye el Indice de Oscilación del Sur IOS, que es la diferencia estandarizada entre las presiones atmosféricas superficiales de ambos sitios.

El fenómeno ENSO tiene dos fases extremas: el Niño (cálido) y la Niña (frío o neutral), que resultan de la interacción entre las superficies del océano y la atmósfera en el Pacífico tropical y se manifiestan a través de grandes cambios de temperatura en la superficie marina. Los cambios en el océano alteran los patrones atmosféricos y climáticos alrededor del mundo y a su vez los cambios en la atmósfera impactan las temperaturas y corrientes del océano. El sistema oscila entre las fases el Niño y la Niña con un promedio de 3 o 4 años por ciclo. Los cambios ocasionados por el ENSO pueden causar inundaciones en algunos sitios y sequías en otros. (NOAA, 2003).

La figura 1 muestra la oleada cálida en cercanías a la costa pacífica colombiana durante la ocurrencia del fenómeno El Niño en 1997.

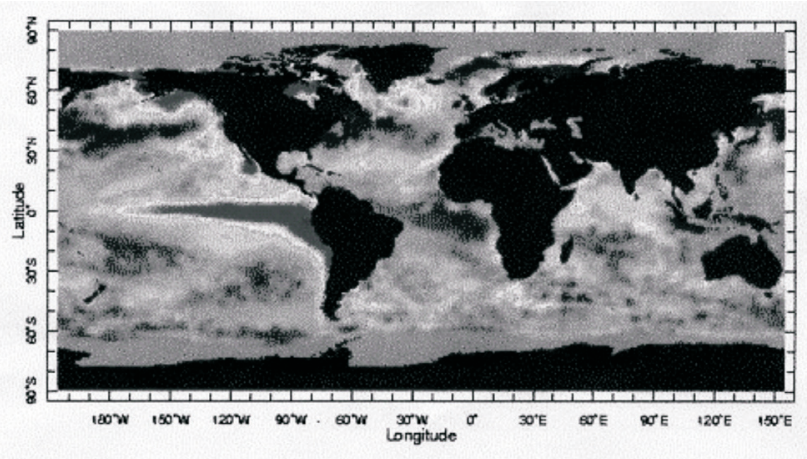

Figura 1. Evento El Niño, Agosto, 1997

Fuente: http://noaa.gov

El cambio en el régimen de lluvias y evaporación han afectado la dinámica y la distribución en el espacio y en el tiempo de la oferta hídrica en términos de cantidad y calidad, ya que cambia también la evapotranspiración, la escorrentía, la infiltración y el almacenamiento subterráneo, por consiguiente los caudales de los ríos que surten la demanda del territorio colombiano.

Durante el evento El Niño 1997-1998, el más fuerte de los últimos años, se presentó un déficit generalizado de lluvias durante casi doce meses. Los niveles y caudales de los ríos en enero de 1998 registraron los valores más bajos de los últimos cincuenta años. La sequía dominó cerca del $90 \%$ del territorio colombiano. De 1160 municipios, 100 presentaron déficit extremo, 861 déficit, 67 déficit ligero y 42 condiciones normales. (IDEAM, 2002).

Según CRID, 2002, una de las áreas más afectadas en cuanto a disminución de caudal es la región del Magdalena, hecho que justifica un estudio más detallado de la cuenca, con el fin de determinar el grado de déficit y las medidas para prevención y mitigación de los problemas que puedan causarse.

\section{B. Oscilación del Atlántico Norte (NAO)}

La Oscilación del Atlántico Norte se caracteriza por- 
que la masa atmosférica se alterna entre las regiones subtropical y subpolar del Océano Atlántico Norte. (Poveda \& Mesa, 1996). Esto ocasiona variaciones en la temperatura superficial del mar a nivel mensual y estacional, cambios en los vientos del Oeste en latitudes medias, diferencias del gradiente de presiones en escala mensual y estacional, y por lo tanto afecta el clima en la región continental adyacente.

Durante la fase positiva de la NAO se producen numerosas tormentas sobre el Océano Atlántico Norte. Dichas tormentas llevan aire caliente con gran cantidad de vapor de agua al Norte de Europa, esto hace que el invierno sea más húmedo y cálido. Más al sur, los vientos llevan aire frio y seco de la tierra a la zona mediterránea haciendo que allí el invierno sea soleado pero frío.

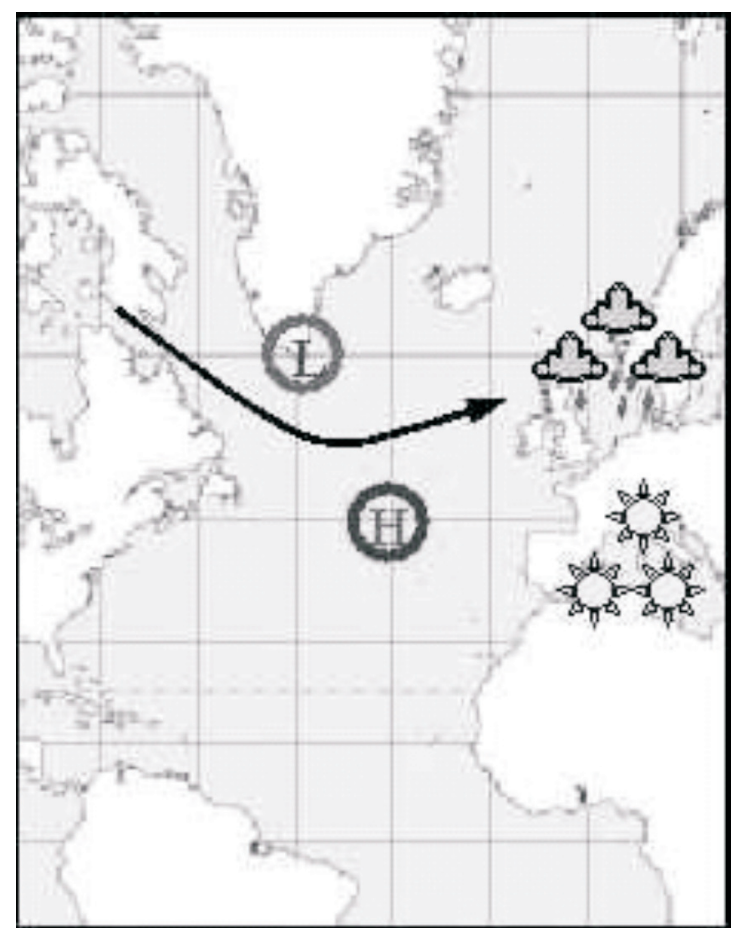

Figura 2. Fase positiva de la NAO

Fuente: http://www-atmosphere.mpg.de/enid/

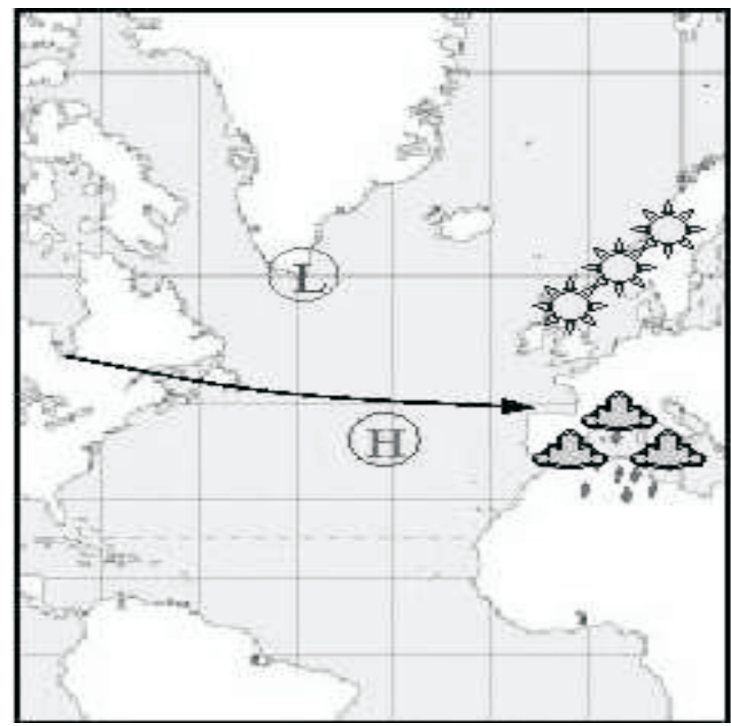

Figura 3. Fase negativa de la $N A O$

Fuente: http://www-atmosphere.mpg.de/enid/

Durante los inviernos de la fase NAO negativa (ver figura 3), hay muchas menos tormentas sobre el Atántico Norte. Estas tormentas llevan aire húmedo y cálido del océano a regiones del Mediterráneo. La pequeña diferencia de presión permite que el aire del norte llegue al Norte de Europa haciendo que los inviernos sean secos y soleados pero fríos.

El fenómeno es comparable con la OS en términos de la variabilidad de presiones en sitios claves. Se mide a través de un índice obtenido mediante la diferencia de presión atmosférica en Punta Delgada (Azores) y Akureyri (Islandia). Y aunque ambos fenómenos influyen en la distribución de la presión atmosférica sobre el Atlántico occidental y el este de Norteamérica, no parece existir una conexión entre ellos (Rogers, 1984, citado por Mesa \& Poveda, 1997). Rogers sugiere que la precipitación promedio sobre el Caribe y la zona tropical de América podría variar significativamente en los eventos extremos de la Oscilación del Atlántico Norte, lo cual evidencia un posible vínculo con la hidrología nacional. 


\section{El cambio climático}

No sólo los fenómenos naturales de origen climático pueden afectar la disponibilidad hídrica. La actividad del hombre, que demanda cada vez más todo tipo de recursos naturales ha comenzando a comprometer el recurso clima en la escala global (IPCC, 1995). Está comprobado que estas actividades están aumentando la concentración de los gases que intensifican el efecto invernadero de la atmósfera (dióxido de carbono, metano, clorofluorocarbonos, óxido nitroso, hidrofluorocarbonos, perfluorocarbonos y hexafluoruro de azufre entre los más importantes).

La temperatura del planeta se ha incrementado en el último siglo en cerca de $0,5^{\circ} \mathrm{C}$, y científicamente se ha comprobado que buena parte de este aumento es atribuible al efecto invernadero de origen antropogénico (IPCC, 1995). Si el ritmo del crecimiento de las emisiones continúa sin ningún tipo de limitación, se estima que para el año 2025 la temperatura media del planeta se incrementaría en $1^{\circ} \mathrm{C}$ y para fines del próximo siglo en $3^{\circ} \mathrm{C}$. Los incrementos de la temperatura no serán homogéneos sobre el planeta y como consecuencia, todo el sistema climático se alteraría, modificando los niveles de precipitación media en muchas regiones e incrementando los eventos extremos generadores de inundaciones y sequías. La figura 4 ilustra los cambios en la tem-peratura a nivel mundial.

Un cambio climático global de la magnitud y velocidad prevista podría provocar alteraciones importantes en la biosfera conduciendo a migraciones y extinciones de numerosas especies y al aumento del nivel del mar. Estos cambios perjudicarían también a las actividades agropecuarias y a la generación hidroeléctrica.

Los modelos climáticos computacionales anticipan que el ciclo de evapotranspiración se acelerará a nivel mundial y esto implica que lloverá más, pero que las lluvias también se evaporarán más rápidamente, volviendo los suelos más secos durante los períodos críticos de la temporada de cultivo (Prodiversitas, 2005). Nuevas sequías, o más intensas, en particular en los países más pobres, podrían disminuir el abastecimiento de agua potable hasta el punto de amenazar la salud pública.

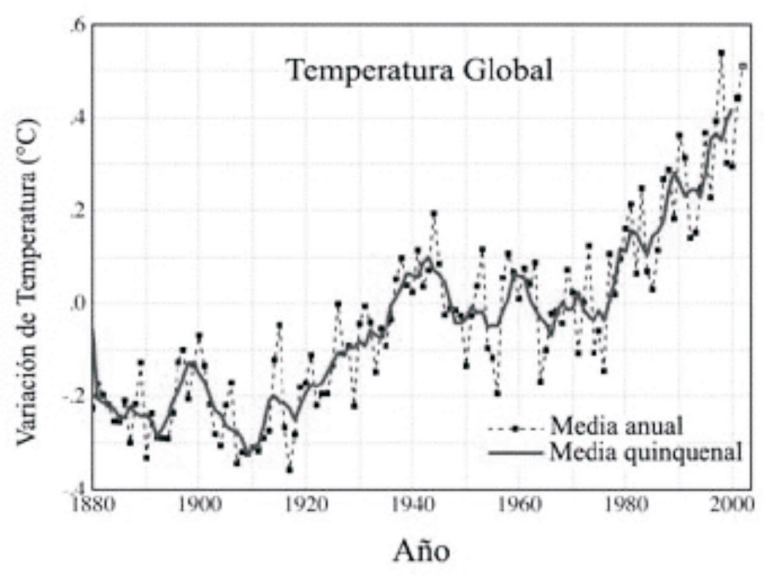

Figura 4. Cambios en la temperatura global desde 1880 Fuente: $h t t p: / / w w w . p r o d i v e r s i t a s . b i o e t i c a . o r g / d e s 41 . h t m$

\section{El Río Magdalena}

El río Magdalena es considerado el más importante del país y el más largo, con una longitud de $1540 \mathrm{Km}$. A lo largo de su curso recibe unos 500 ríos, numerosas quebradas y registra en la estación Calamar, la más cercana a la desembocadura, un caudal promedio de $8.224 \mathrm{~m}^{3} / \mathrm{s}$ para el periodo de registro entre 1969 y 1999. Nace en la laguna de La Magdalena, $1^{\circ} 56^{\prime} \mathrm{N}, 76^{\circ} 35^{\prime} \mathrm{O}$, en el páramo de las Papas, a 3685 m.s.n.m y corre a lo largo de los Andes hasta desembocar en Bocas de Ceniza en el Mar Caribe. El río en su mayor parte es poco profundo y tortuoso y la deforestación ha causado aluviones que han reducido su navegabilidad. Hoy solo un tramo final es navegable (IGAC, 1996).

El Magdalena sirve para muchos propósitos, tanto para extraer agua como para verter residuos de los 
sistemas de alcantarillado en la mayoría de ciudades colombianas. En menor escala es aprovechable para la navegación fluvial, la pesca y la generación de energía hidroeléctrica (Sarmiento, 2004).

La cuenca de drenaje del Magdalena tiene un área de $257.000 \mathrm{~km}^{2}$ y representa cerca del $25 \%$ del área continental del país. Su red hidrográfica abastece las necesidades hídricas de más de la mitad de la población colombiana asentada en la cuenca, donde se genera aproximadamente el $85 \%$ del PIB nacional. Su densidad de población es equivalente a 120 habitantes/ Km² (Colciencias, 1999).

De acuerdo con sus características de navegabilidad el río se ha subdividido en tres cuencas: alta, media y baja. El área de investigación del presente trabajo corresponde a las cuencas alta y media del Río Magdalena. La cuenca alta comprende desde el nacimiento del río en el Páramo de las Papas, hasta Honda en sentido latitudinal, y en sentido longitudinal, de Este a Oeste, desde las cimas de la Cordillera Central hasta las de la Cordillera oriental, recorre desde su nacimiento $565 \mathrm{Km}$ hasta el salto de Honda, donde interrumpe su paso. La cuenca media va desde Honda hasta el municipio de Morales en Bolívar, sin embargo para efectos del presente estudio la cuenca media se tomó hasta el municipio de Barrancabermeja. Por último el tramo conocido como cuenca baja va de Morales hasta Barranquilla y Cartagena (IGAC, 1996). A continuación se presentan algunas características importantes tomadas de (IGAC, 1996) y (Alvear, 2005).

\section{Caracterización de la Cuenca Alta}

La región del Valle del Alto Magdalena posee todos los pisos térmicos. En la región plana la temperatura aumenta hacia el norte y en la medida en que disminuye la altitud. La temperatura regional varía entre un poco menos de siete grados hasta valores superiores a 23 grados, en promedio.
El régimen de lluvias desde el nacimiento del río hasta San Agustín es monomodal entre los meses de marzo y septiembre, que alcanza su valor máximo en el mes de junio. De ahí en adelante la distribución temporal es de tipo bimodal, caracterizada por tener dos épocas lluviosas y dos épocas secas en el año. La primera temporada lluviosa es más intensa que la segunda y se presenta entre marzo y junio, con valores máximos en abril y mayo. La segunda temporada lluviosa va de septiembre a mediados de diciembre. El primer periodo seco se presenta entre mediados de diciembre y comienzos de marzo y el segundo entre finales de junio y comienzos de septiembre.

La distribución espacial de la precipitación presenta un rango de variación que va desde los $1000 \mathrm{~mm}$ a valores superiores a los $3500 \mathrm{~mm}$ en promedio al año.

La región comprendida entre las ciudades de Neiva y Girardot se extiende a través de los llanos secos del Huila y del Tolima, valles y montañas del río Saldaña y la vertiente al Magdalena de la Cordillera Oriental colindante con el páramo de Sumapaz. Primero el río recorre un valle estrecho, que se amplía hacia su margen izquierda a la altura de Natagaima, mientras que en la derecha el valle no supera en ningún punto los 25 kilómetros de anchura. El río Magdalena recibe en esta subregión, numerosos afluentes entre ellos el Baché, Aipe, Saldaña, Coello, Las Ceibas, Ioro, Fortalecillas, Bateas, Villa-vieja, Cabrera, Yaví, Cunday y Sumapaz.

Entre la desembocadura del Río Bogotá y los raudales de Honda, el río recorre $145 \mathrm{Km}$, con un desnivel de 69 metros entre los dos puntos. Se trata de un valle intercordillerano estrecho, puesto que a esta altura no sobrepasa los 40 kilómetros de anchura. Presenta, sin embargo, diferentes características sobre cada una de sus bandas, a la izquierda es bastante regular, con una anchura promedio de 20 kilómetros; mientras que hacia la margen derecha se estrecha 
sensiblemente a partir de la desembocadura del río Seco, presentando numerosas digitaciones y colinas bajas que mueren a menos de 10 kilómetros del río. Desembocan al Magdalena los siguientes tributarios sobre la margen izquierda: Río Coello, Río Totaré, quebradas Agua Blanca, Tantan, La Pena, ríos Lagunilla y Guamo, Quebrada Seca y río Gualí; sobre la margen derecha, los ríos Bogotá, Seco, Seco de Palmas y varias quebradas y arroyos menores.

Los suelos son altamente productivos, por tratarse de llanuras aluviales, así como de abanicos aluviales provenientes especialmente de la Cordillera Central. La vegetación predominante hasta los 500 m.s.n.m, que representa las tres cuartas partes de la extensión de este sector, corresponde al bosque seco tropical; por encima de los 500 m.s.n.m. y hasta los 1.000 m.s.n.m. predomina el bosque húmedo premontano.

\section{Caracterización de la Cuenca Media}

Se denomina Valle Intermedio del río Magdalena al área comprendida entre la ciudad de Honda y el municipio de Morales, en el departamento de Bolívar. El río en esta región recorre $260 \mathrm{Km}$, y alcanza una diferencia en alturas entre los dos puntos de $145 \mathrm{~m}$.

El valle en esta región presenta diferencias entre su margen izquierda perteneciente a la cordillera central y su margen derecha que corresponde a la cordillera Oriental. Sobre la margen izquierda el valle conserva un ancho promedio de $30 \mathrm{Km}$ desde la desembocadura del río Guarinó, en límites entre los departamentos de Tolima y Caldas. Más adelante, ya en territorio antioqueño, el valle presenta un perfil sinuoso sobre las estribaciones de la Cordillera Central, en donde su ancho en promedio varía entre 35 y 40 kilómetros.

Sobre la margen derecha, a la altura de los Saltos de Honda, el valle alcanza apenas 10 kilómetros de ancho sobre la cota de los 1.000 m.s.n.m., pero más al norte se va abriendo poco a poco en las cabeceras del río Negro en cercanías de Puerto Salgar, para ampliarse definitivamente en el Territorio Vásquez, alcanzando posteriormente, a la altura de Barrancabermeja, los 60 kilómetros de ancho. En esta banda el Magdalena recibe el tributo de los siguientes cursos de agua: ríos Negro, Palenque, Hermitaño, y Quebradas La Muerta, Carolina y Montoyas, ríos Carare y Opón.

Los suelos son variables en fertilidad y capacidad de uso. La vegetación predominantemente es el bosque húmedo tropical y el bosque húmedo premontano; posee una alta precipitación y clima cálido con más de $26^{\circ} \mathrm{C}$.

La climatología es de régimen bimodal, caracterizada por una temporada seca en los meses de diciembremarzo y julio-septiembre y una temporada lluviosa durante los meses de abril-mayo y octubre-noviembre. Sin embargo en los últimos años, se ha notado una amplia variación del clima en el territorio debido a fenómenos macroclimáticos y de calentamiento global.

\section{INFORMACIÓN HIDROLÓGICA}

Las series de datos para el presente estudio fueron suministrados por el IDEAM a través del convenio suscrito con la Universidad de Los Andes. Las estaciones se escogieron teniendo en cuenta varios criterios: que las series de tiempo fueran continuas, es decir que no tuvieran lagunas superiores a 3 años consecutivos, se prefirieron las estaciones con mayor número de registros, es decir las instaladas antes de 1990 y que no hubieran sido suspendidas.

La información correspondiente a las es-taciones de Paso del Colegio, Los Cuartos, Puerto Imarco y Barrancabermeja no se utilizó en este estudio, debido a que presenta menos de 8 años de registro. En total se cuenta con 40 estaciones y aunque cuatro de ellas poseen series cortas de registros cercanas a los 
12 años se contemplaron con el fin de hacer comparaciones (ver Tabla 1). La información se procesó a partir de los datos existentes, sin realizar modificaciones a las series, con el fin de obtener pronósticos más cercanos a la realidad. Además se aprovechó el hecho de que se tienen programas robustos de análisis no paramétrico, que admiten datos perdidos y no exigen una determinada distribución de éstos.

Para confirmar la homogeneidad de los datos se realizaron varias pruebas. Inicialmente se graficaron los datos con el fin de observar visualmente los valores por fuera del rango normal y analizar posibles errores de digitación; también se compararon con estaciones vecinas, teniendo en cuenta que un dato alto en al-

Tabla 1. Estaciones del Alto y Medio Magdalena

\begin{tabular}{|c|c|c|c|}
\hline Río, Estación, Dpto & cota & $\begin{array}{l}\text { Periodo } \\
\text { registro }\end{array}$ & $\begin{array}{l}\text { Años } \\
\text { comp }\end{array}$ \\
\hline 1 Magdalena, Cascada S. B,. Huila. & 1190 & 1964-1994 & 31 \\
\hline 2 Magdalena, Salado Blanco, Huila & 1070 & $1964-2003$ & 40 \\
\hline 3 Magdalena, La Magdalena, Huila & 1700 & $1991-2003$ & 11 \\
\hline 4 Magdalena, Pericongo, Huila & 950 & $1964-2003$ & 40 \\
\hline 5 Suaza, Puente Garcés, Huila & 970 & $1964-2003$ & 38 \\
\hline 6 Magdalena, Pte Balseadero, Huila & 680 & $1965-2003$ & 39 \\
\hline 7 Páez, Paicol, Huila & 785 & $1964-2003$ & 38 \\
\hline 8 Magdalena, Vichecito, Huila & 620 & 1985-1994 & 10 \\
\hline 9 Yaguará, Hda Jardín, Huila & 585 & $1976-2003$ & 28 \\
\hline 10 Yaguará, Hda Venecia, Huila & 575 & $1983-2003$ & 21 \\
\hline 11 Magdalena, Pte Santander, Huila & 430 & $1948-2003$ & 47 \\
\hline 12 Magdalena, La Esperanza, Huila & 460 & $1994-2003$ & 10 \\
\hline 13 Neiva, Pte Mulas, Huila & 740 & $1967-2003$ & 37 \\
\hline 14 Villavieja, Polonia, Huila & 598 & $1965-1988$ & 12 \\
\hline 15 Magdalena, Purificación, Tolima & 297 & $1959-2002$ & 44 \\
\hline 16 Chenche, Purificación 1, Tolima & 310 & $1965-2002$ & 38 \\
\hline 17 Aipe, Pte Carretera, Huila & 385 & $1972-2003$ & 32 \\
\hline 18 Magdalena, Angostura, Tolima & 345 & $1959-2003$ & 45 \\
\hline 19 Cabrera, San Alfonso, Huila & 420 & $1959-2003$ & 45 \\
\hline 20 Prado, Boquerón, Tolima & 340 & $1959-2002$ & 43 \\
\hline 21 Sumapaz, El Limonar, Tolima & 405 & $1965-2002$ & 38 \\
\hline 22 Coello, Payandé, Tolima & 560 & $1983-2002$ & 20 \\
\hline 23 Opía, Piedras, Tolima & 350 & $1973-2002$ & 30 \\
\hline 24 Magdalena, Nariño, Cundinamarca & 300 & $1934-2002$ & 67 \\
\hline 25 Magdalena, Arrancaplumas, Cundin. & 230 & $1934-2002$ & 68 \\
\hline 26 Seco, Corralitos, Cundinamarca & 704 & $1952-2002$ & 50 \\
\hline 27 Totaré, Bocatoma, Tolima & 420 & $1976-2002$ & 27 \\
\hline 28 Sabandija, Pte San Fco, Tolima & 260 & $1963-2002$ & 40 \\
\hline 29 Lagunilla, La Esmeralda, Tolima & 210 & $1988-2002$ & 15 \\
\hline 30 Recio, La Nueva, Tolima & 470 & $1960-2002$ & 43 \\
\hline 31 Gualí, Pte López, T & 194 & $1971-2002$ & 32 \\
\hline 32 La Miel, San Miguel, Antioquia & 160 & $1965-2002$ & 38 \\
\hline 33 Nare, Canteras, Antioquia & 127 & $1965-2002$ & 38 \\
\hline 34 Magdalena, Pto Berrío, Antioquia & 108 & $1936-2003$ & 68 \\
\hline 35 Magdalena,Peñas Blancas, Antioquia & 80 & $1977-2003$ & 20 \\
\hline 36 Negro, Pto Libre, Boyacá & 154 & $1965-2002$ & 38 \\
\hline 37 Magdalena, Pto Salgar, Cundin. & 168 & $1937-2002$ & 57 \\
\hline 38 Negro, Colorados, Cundinamarca & 286 & $1952-2002$ & 50 \\
\hline 39 Carare, Pto Araújo, Santander & 92 & $1965-2003$ & 39 \\
\hline 40 Magdalena, Maldonado, Santander & 86 & $1979-2001$ & 22 \\
\hline
\end{tabular}

guna serie debe corresponderse espacialmente con las estaciones cercanas.

Además se realizó el cálculo de estadísticos básicos tales como la media, la varianza, la desviación estándar, pruebas T, pruebas de Fisher, autocorrelaciones, correlación cruzada y tendencia por medio de la prueba estadística de Mann-Kendall.

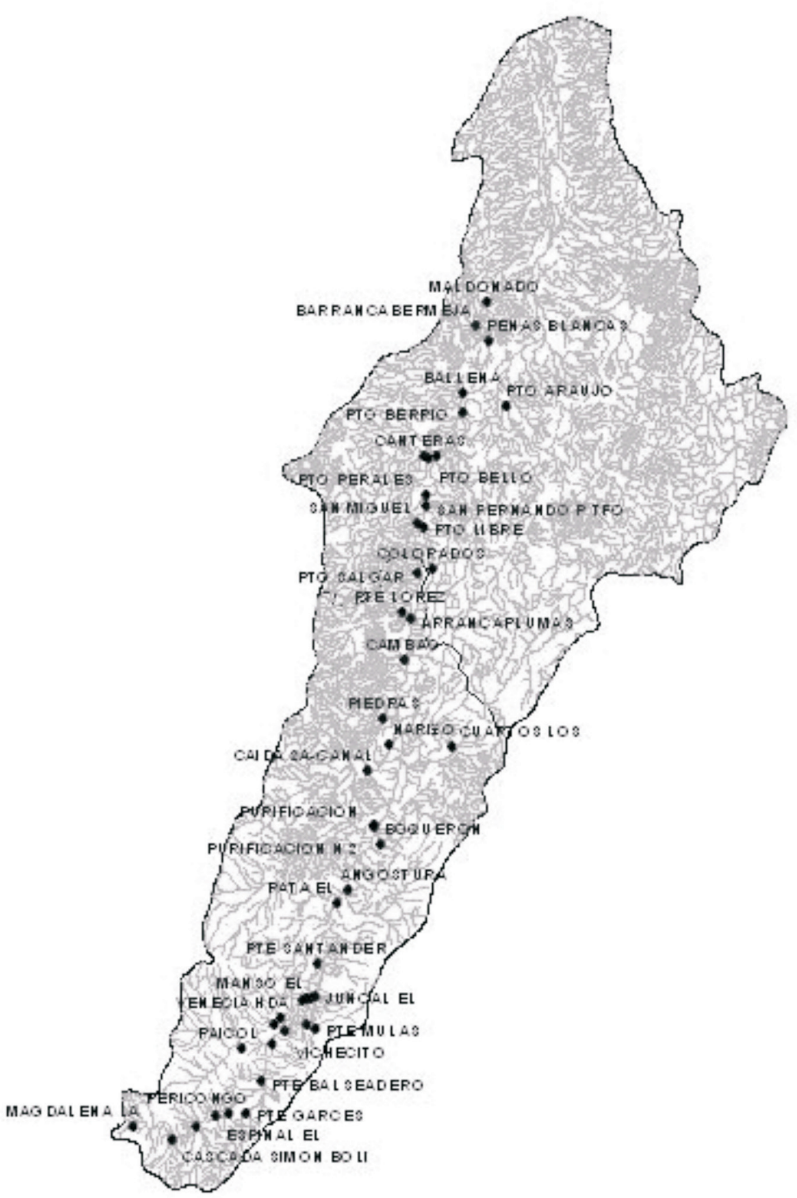

Figura 5. Localización de las estaciones Alto y Medio Magdalena

De un total de 40 estaciones, 16 se sitúan sobre el Río Magdalena, dos sobre el Río Yaguará y dos sobre el Río Negro. Las 20 estaciones restantes se encuentran ubicadas sobre los ríos Suaza, Páez, Neiva, Villavieja, Chenche, Aipe, La Cabrera, Sumapaz, Prado, Coello, 
Opía, Seco, Totaré, Sabandija, Lagunilla, Recio, Gualí, La Miel, Nare y Carare.

En cuanto a la distribución departamental, 16 estaciones pertenecen al Huila, 12 al Tolima, 5 a Cundinamarca, 1 a Boyacá, 4 a Antioquia y 2 a Santander.

Con el fin de facilitar la ubicación espacial de las estaciones de trabajo, se utilizó un shape o mapa digital que permite su digitalización georeferenciada. En la figura 5 se presenta la localización de las estaciones.

\section{ANÁLISIS ESTADÍSTICO}

En primera instancia se determinaron las estaciones que servirían para el estudio de acuerdo con la información dada anteriormente. Así mismo se empleó el programa Dissman para transformar las coordenadas y se procedió a completar los datos que fueran posibles a través del programa Chac (Cedex, 2004), que permite realizar correlaciones multivariadas entre grupos de estaciones cercanas y que posean el mismo comportamiento climático. La única estación que se complementó fue la de Puente Garcés, las demás estaciones se procesaron con todos sus datos faltantes a través de los programas Makesens para la determinación de tendencias y Mann-Kendall para la determinación de correlaciones. Estos cálculos se realizaron también a través de Excel y del programa SPSS con el fin de corroborar los resultados.

El Test de Mann-Kendall, empleado en los dos programas mencionados anteriormente se considera robusto, ya que es no paramétrico, se puede utilizar para evidenciar la existencia de una tendencia en la serie, no tiene una complejidad matemática excesiva y rechaza calificar una serie como estadísticamente significativa a no ser que ésta permanezca hasta la finalización del periodo de estudio. Esto evita que puedan aparecer subperiodos cortos que se confundan con una tendencia de toda la serie. Es un test potente, ya que indica que la probabilidad de rechazar la hipótesis nula cuando existe una tendencia es tan alta como la que daría un test paramétrico, además no es preciso conocer la distribución de los datos de la serie analizada.

El análisis de correlación se realizó mediante la aplicación del test de Mann-Kendall y se confirmó mediante cálculos en hoja Excel y a través del programa SPSS. Se estableció la correlación entre la serie de datos de caudales Vs los índices MEI y NAO, debido a que las investigaciones anteriores han demostrado que son los de mayor incidencia en las variaciones climáticas de la zona en estudio (Poveda, 2005).

\section{A. Correlación con el índice MEI}

Los resultados para las estaciones que presentan algún grado de correlación negativa con el índice MEI se muestran en la tabla 2.

En principio podría causar alarma que de 40 estaciones, 26 estén presentando algún grado de correlación negativa con el fenómeno ENSO (mientras el ENSO se intensifica, el caudal disminuye, ver figura 6). Esto quiere decir que el índice MEl explica en parte la ocurrencia de disminuciones de caudal.

Sin embargo, es necesario mirar con mucho cuidado estos resultados, teniendo en cuenta que solamente cinco de ellos poseen un nivel de confianza mayor al $90 \%$. Esto sugiere que para las estaciones de Puente Garcés que pertenece al Río Suaza, Puente Santander, Purificación y Angostura, que pertenecen a la corriente del Río Magdalena y Puente Mulas del Rio Neiva, la disminución de caudal sí se encuentra influenciada en buena parte por el fenómeno del Niño, mientras que para las otras la disminución en el caudal puede explicarse por otros fenómenos tales como el crecimiento de la población y el aprovechamiento del recurso hídrico para riego, acueduc-tos, hidroeléctricas, consumo humano, etc (Poveda, Mesa y Carvajal, 1997). 
Tabla 2. Estaciones que presentaron correlación negativa con índice MEI para más de tres meses

\begin{tabular}{|c|c|c|c|c|c|c|c|c|c|}
\hline & & & Corl & relac & ción & & & & \\
\hline Nombre & E F & M & A M & $\mathrm{J} \quad \mathrm{J}$ & $\mathrm{A}$ & $\mathrm{S} O$ & $\mathrm{~N} \mathrm{D}$ & D P & P-value \\
\hline 1 Cascada Simón B & & & & & & & & & 0,202 \\
\hline 2 Salado Blanco & & & & & & & & & 0,232 \\
\hline 4 Pericongo & & & & & & & & & 0,364 \\
\hline 5 Puente Garcés* & & & & & & & & & 0,099 \\
\hline 6 Pte Balseadero & & & & & & & & & 0,247 \\
\hline 7 Paicol & & & & & & & & & 0,463 \\
\hline 8 Vichecito & & & & & & & & & 0,329 \\
\hline 11 Pte Santander* & & & & & & & & & 0,000 \\
\hline 13 Pte Mulas* & & & & & & & & & 0,099 \\
\hline 15 Purificación* & & & & & & & & & 0,026 \\
\hline 18 Angostura* & & & & & & & & & 0,000 \\
\hline 22 Payandé & & & & & & & & & 0,282 \\
\hline 25 Nariño & & & & & & & & & 0,138 \\
\hline 26 Arrancaplumas & & & & & & & & & 0,255 \\
\hline 26 Corralitos & & & & & & & & & 0,164 \\
\hline 28 Bocatoma & & & & & & & & & 0,227 \\
\hline 29 Esmeralda La & & & & & & & & & 0,155 \\
\hline 31 Pte López & & & & & & & & & 0,119 \\
\hline 32 San Miguel & & & & & & & & & 0,198 \\
\hline 33 Canteras & & & & & & & & & 0,493 \\
\hline 34 Pto Berrío & & & & & & & & & 0,156 \\
\hline 36 Pto Libre & & & & & & & & & 0,217 \\
\hline 37 Pto Salgar & & & & & & & & & 0,325 \\
\hline 38 Colorados & & & & & & & & & 0,402 \\
\hline 39 Pto Araújo & & & & & & & & & 0,118 \\
\hline 40 Maldonado & & & & & & & & & 0,392 \\
\hline
\end{tabular}

Todas las estaciones muestran correlación negativa con el fenómeno ENSO al menos durante los tres primeros meses del año, lo cual evidencia que mientras el fenómeno El Niño va en aumento, se presenta disminución de los caudales. En la tabla 3 figuran las estaciones que no presentaron correlación.

Tabla 3. Estaciones que no presentaron correlación con el indice MEI

\begin{tabular}{|rlrrrr|}
\hline No. & Nombre & & No. & Nombre & p-value \\
\hline 3 & Magdalena La & 0,491 & 20 & Boquerón & 0,021 \\
9 & Jardín El Hda. & 0,227 & 21 & Limonar El & $* 0,031$ \\
10 & Venecia Hda & 0,181 & 22 & Cuartos Los & $* 0,026$ \\
12 & Esperanza La & 0,45951 & 24 & Piedras & 0,299 \\
14 & Polonia & 0,10616 & 29 & Pte San Fco. & 0,228 \\
16 & Purificación 1 & 0,2294 & 31 & Nueva La & 0,385 \\
17 & Pte Carretera & 0,11004 & 36 & Peñas Blancas & $* 0,087$ \\
19 & San Alfonso & $* 0,07844$ & 40 & Puerto Araújo & 0.118 \\
\hline
\end{tabular}

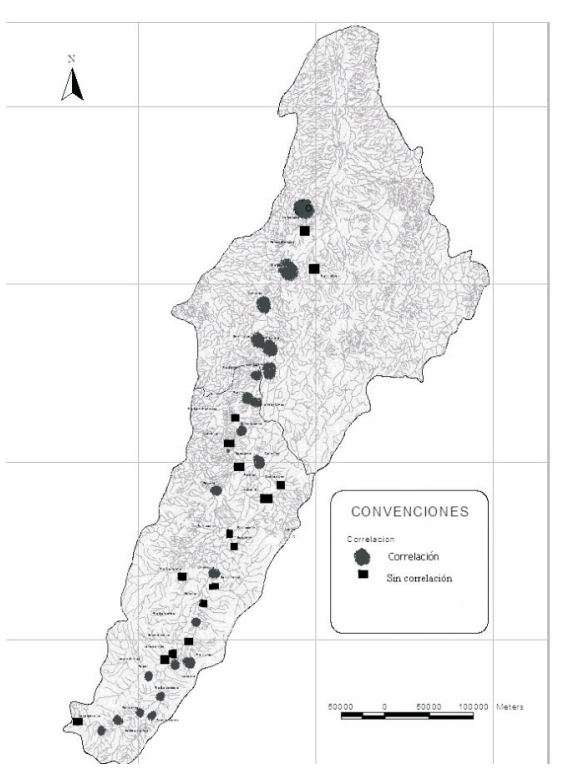

Figura 6. Correlación caudal vs MEI

Las figuras 7, 8, 9 y 10 muestran los valores de correlación trimestrales para cada estación. Las estaciones se presentan en orden iniciando por la primera estación de la cuenca Alta: Cascada Simón Bolívar y terminando con la última de la cuenca Media: Maldonado. En la figura 7 para el primer trimestre, se observan todos los valores de correlación (por debajo de -0.1) para cada una de las estaciones.

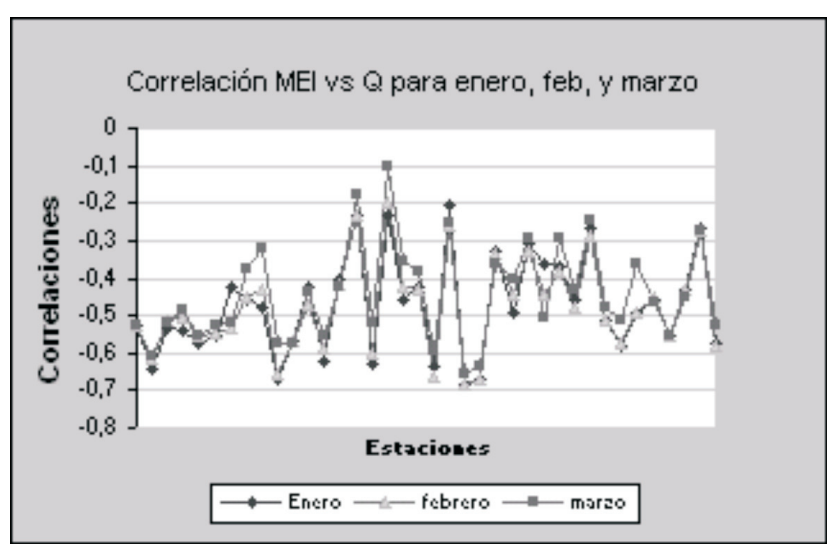

Figura 7. Correlación MEI vs. Q para el primer trimestre 


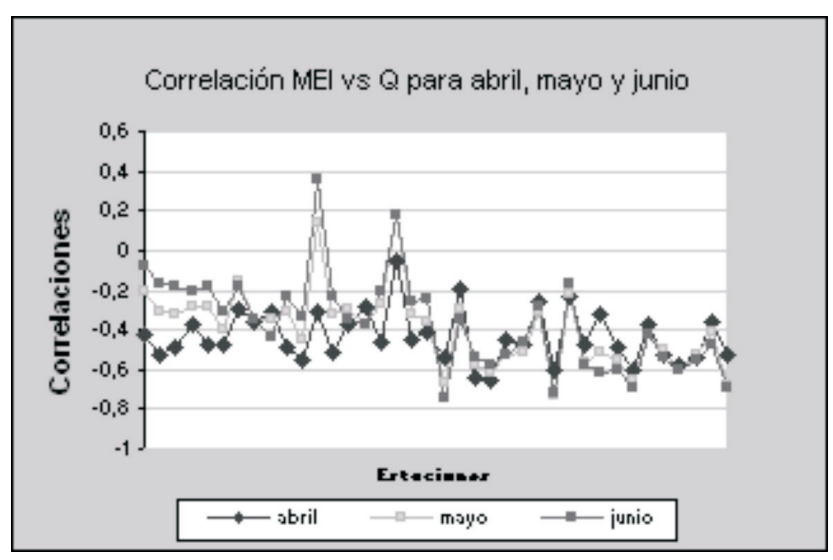

Figura 8. Correlación MEI vs. Q para el segundo trimestre

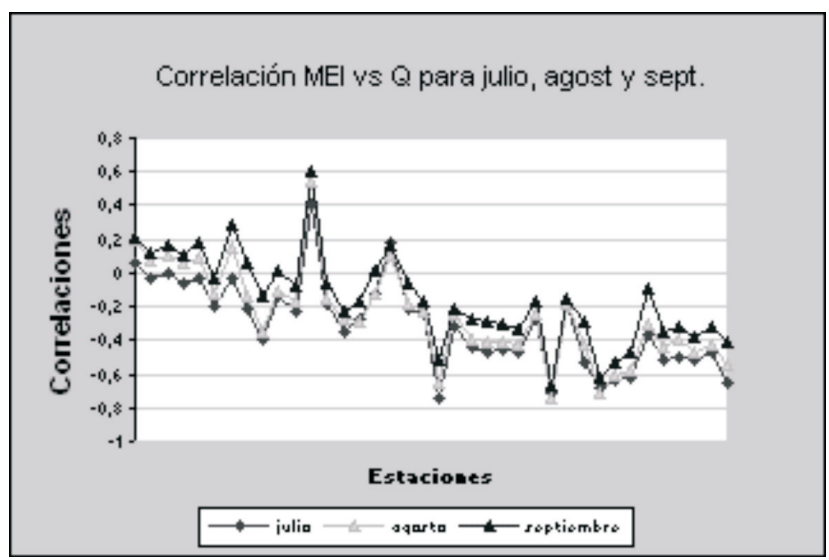

Figura 9. Correlación MEI vs. Q para el tercer trimestre

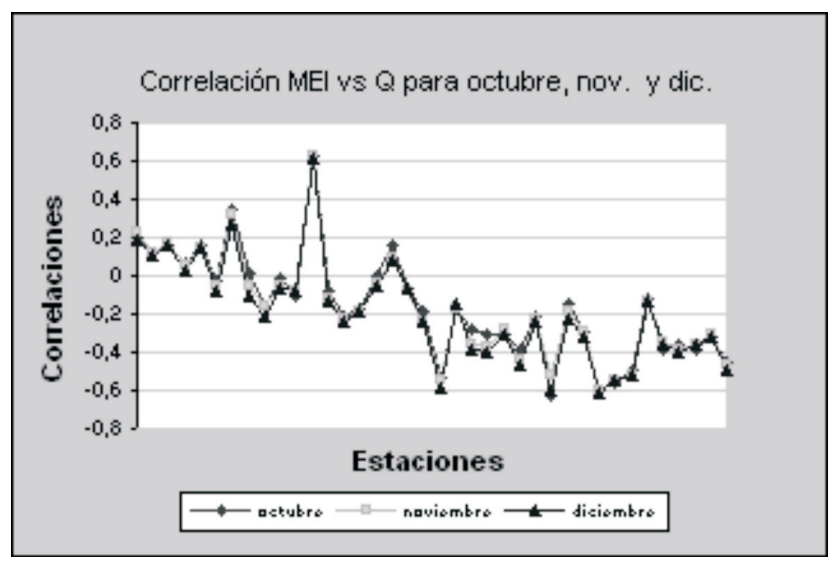

Figura 10. Correlación MEI vs. Q para el último trimestre
Para el segundo trimestre solamente las estaciones de Polonia y San Alfonso presentan correlaciones positivas. Esto evidencia todavía una alta influencia del fenómeno El Niño en la disminución de caudales de la zona (Ver figura 8).

Para el tercer trimestre aproximadamente la mitad de las estaciones presentan correlación positiva, aunque no muy significativa, mientras que la otra mitad sigue presentando correlación negativa (Ver figura 9).

Para los últimos meses del año aproximadamente un $78 \%$ de las estaciones presentan correlación negativa (Ver figura 10).

\section{Tabla 4. Correlación NAO vs. Caudal}

Estaciones Ene Feb Mar Abr May Jun Jul Ago Sep Oct Nov Dic pval

1 Cascada Simòn B0,03-0,27-0,06-0,02-0,36 0,39 0,04 0,14 0,00-0,07-0,02 -0,04 0,05

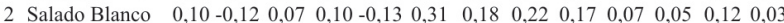

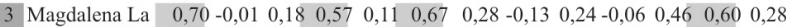

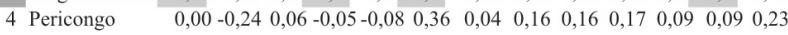

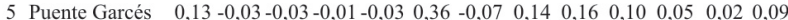

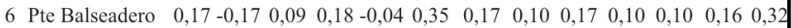

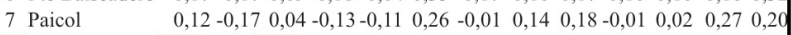

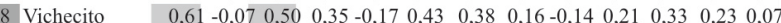

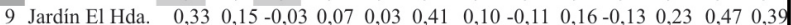

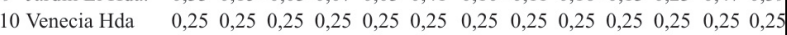

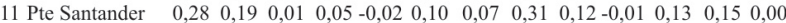

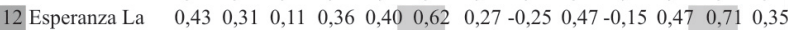
13 Pte Mulas 14 Polonia

15 Purificación 16 Purificación 1

17 Pte Carretera

18 Angostura

19 San Alfonso

20 Boquerón

21 Limonar El

22 Payandé

23 Piedras

24 Nariño $\begin{array}{llllllllll}-0,15-0,05 & -0,01-0,14-0,15 & 0,25 & 0,11 & 0,05 & 0,27 & 0,13 & 0,00 & 0,12 & 0,22\end{array}$

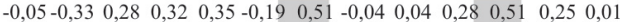
$\begin{array}{lllllllllllll}0,26 & 0,16 & 0,08 & 0,05 & 0,00 & 0,15 & 0,07 & 0,28 & 0,18 & -0,02 & 0,19 & 0,31 & 0,04\end{array}$ $\begin{array}{llllllllll}0,11-0,18-0,14-0,06-0,01 & 0,13 & 0,00 & 0,00-0,18-0,13 & 0,01 & -0,03 & 0,04\end{array}$

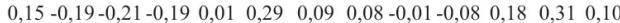
$\begin{array}{llllllllllllllll}0,34 & 0,21 & 0,02 & 0,05 & 0,00 & 0,08 & 0,07 & 0,31 & 0,14 & -0,04 & 0,21 & 0,20 & 0,00\end{array}$ $\begin{array}{llllllllllllll}0,28 & 0,29 & 0,08 & -0,04 & -0,01 & -0,04 & 0,11 & 0,32 & 0,18 & 0,10 & 0,20 & 0,23 & 0,15\end{array}$

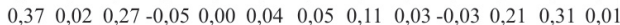
$\begin{array}{lllllllllllll}0,41 & 0,15 & 0,06 & -0,06 & -0,07 & -0,03 & -0,06 & 0,16 & -0,12 & -0,03 & 0,05 & 0,21 & 0,03\end{array}$

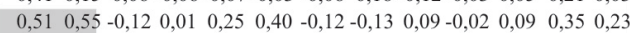

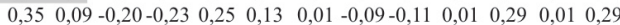

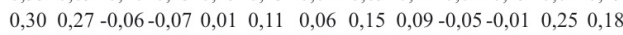

25 Arrancaplumas $0,29 \quad 0,23-0,08-0,12 \quad 0,04 \quad 0,14 \quad 0,04 \quad 0,16 \quad 0,08 \quad 0,00 \quad 0,01 \quad 0,22 \quad 0,13$

26 Corralitos $\quad-0,15-0,25-0,28-0,02 \quad 0,06 \quad 0,13-0,01-0,200,23 \quad 0,11-0,03 \quad 0,18 \quad 0,05$

27 Bocatoma $\quad 0,43 \quad 0,28 \quad 0,21-0,34-0,14 \quad 0,23 \quad-0,09 \quad 0,23-0,02 \quad 0,28-0,19 \quad 0,08 \quad 0,32$ 28 Pte San Francisc $0,03 \quad 0,08-0,190,03 \quad 0,20-0,05 \quad 0,18-0,12-0,06 \quad 0,10 \quad 0,09-0,160,22$

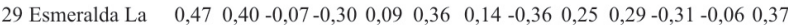

30 Nueva La $\quad 0,210,34 \quad 0,03-0,08-0,13-0,12-0,06 \quad 0,16-0,16-0,05-0,23 \quad 0,180,36$

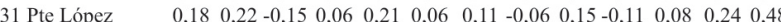

32 San Miguel $\quad 0,24 \quad 0,33-0,19-0,17 \quad 0,33-0,03 \quad 0,00 \quad 0,02 \quad 0,09-0,02 \quad 0,03 \quad 0,02 \quad 0,48$

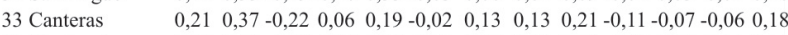

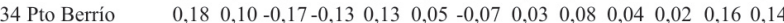

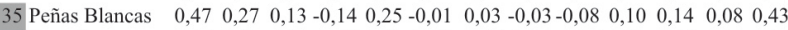

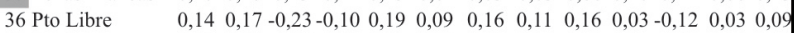

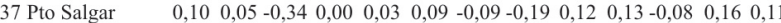

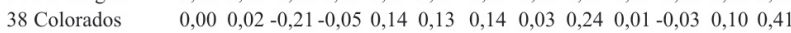

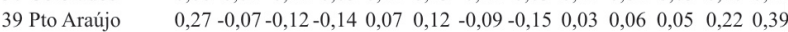

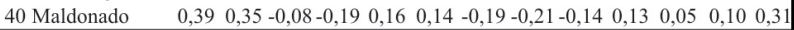


Con el fin de cuantificar el grado de dependencia lineal entre los índices MEI y NAO y los caudales del Río Magdalena, se obtuvieron los coeficientes de correlación de las series totales a diferentes rezagos en el tiempo.

Con relación al índice $\mathrm{MEI}$, las correlaciones muestran valores entre -0.15 y -0.505 , para rezagos negativos en promedio de tres meses. Las series más largas y por lo tanto las más confiables, presentan mayores valores de correlación, lo cual evidencia alta influencia del MEI en las series de caudales. El sector más afectado (es decir el que presenta mayor correlación) va de la estación Arrancaplumas a la estación Puerto Salgar, correspondiente al Medio Magdalena. La cuenca Alta presenta poca influencia entre las primeras ocho estaciones: Cascada y Vichecito con valores de correlación de -0.2, pero a partir del Río Yaguará, en la estación Hacienda El Jardín, los valores se incrementan de -0.3 a -0.38 en la Estación Nariño.

\section{B. Correlación con el índice de Oscilación del Atlántico Norte (NAO)}

La correlación con el índice de Oscilación del Atlántico Norte se realizó al igual que para el índice MEI, a través del programa Mann-Kendall y sus resultados fueron corroborados mediante el programa SPSS y cálculos en hoja Excel.

Se aprecia una escasa correlación con el índice NAO, ya que sólo cinco de las 40 estaciones presentan algún grado de correlación positivo entre 0.5 y 0.7 para diferentes meses del año (se señalan en la tabla 4 en color gris claro) y a excepción de la estación Payandé, las estaciones que presentaron mayor correlación corresponden a series de tiempo cortas, de unos 9 años (señaladas sobre su número de orden con color gris oscuro), estas series cortas no demuestran el real comportamiento de la zona.

El análisis coincide con el trabajo de Poveda, Mesa y Carvajal (1977), en el que afirman que el índice NAO presenta correlaciones altas (negativas) en la costa Atlántica, mientras que hacia el interior las correlaciones son positivas, al parecer debido a la intensidad de los vientos alisios.

Tabla 5. Rezagos (meses) y valores del mayor coeficiente de correlación cruzada entre índices MEI y NAO vs. Caudales

\begin{tabular}{|rlcccc|}
\hline No. & Estación & \multicolumn{3}{c}{ MEI } & \multicolumn{2}{c|}{ NAO } \\
\hline 1 & Cascada S. Bolívar & -8 & $-0,207$ & 11 & $-0,097$ \\
2 & Salado Blanco & -7 & $-0,247$ & 11 & $-0,08$ \\
3 La Magdalena & -12 & 0,201 & 7 & 0,274 \\
4 Pericongo & -7 & $-0,215$ & 8 & 0,076 \\
5 & Puente Garcés & -8 & $-0,225$ & -6 & 0,086 \\
6 Pte Balseadero & -6 & $-0,232$ & -5 & $-0,093$ \\
7 Paicol & -5 & $-0,226$ & 8 & 0,086 \\
8 Vichecito & -8 & $-0,202$ & -4 & 0,231 \\
9 & Jardín EI Hda. & -3 & $-0,309$ & 0 & 0,21 \\
10 & Venecia Hda & -3 & $-0,339$ & 0 & 0,212 \\
11 & Pte Santander & -4 & $-0,294$ & 0 & 0,77 \\
12 & Esperanza La & -5 & $-0,372$ & 8 & 0,334 \\
13 & Pte Mulas & -7 & $-0,367$ & 11 & $-0,122$ \\
14 & Polonia & -4 & $-0,159$ & -5 & 0,232 \\
15 & Purificación & -3 & $-0,35$ & 0 & 0,106 \\
16 & Purificación 1 & -2 & $-0,256$ & 0 & 0,08 \\
17 & Pte Carretera & -4 & $-0,296$ & 0 & 0,119 \\
18 & Angostura & -4 & $-0,302$ & 0 & 0,085 \\
19 & San Alfonso & 12 & 0,15 & 7 & 0,114 \\
20 & Boquerón & -2 & $-0,296$ & 0 & 0,076 \\
21 & Limonar El & -3 & $-0,229$ & 0 & 0,077 \\
22 & Payandé & 2 & $-0,48$ & 8 & 0,179 \\
23 & Piedras & -2 & $-0,163$ & 7 & 0,116 \\
24 & Nariño & -3 & $-0,384$ & 7 & 0,084 \\
25 & Arrancaplumas & -3 & $-0,417$ & 7 & 0,075 \\
26 & Corralitos & -3 & $-0,325$ & -12 & 0,074 \\
27 & Bocatoma & -3 & $-0,288$ & 12 & $-0,137$ \\
28 & Pte San Francisco & -3 & $-0,156$ & 12 & $-0,158$ \\
29 & Esmeralda La & -2 & $-0,505$ & 3 & 0,172 \\
30 & Nueva La & -3 & $-0,149$ & -1 & 0,076 \\
31 & Pte López & -2 & $-0,342$ & 3 & 0,147 \\
32 & San Miguel & -1 & $-0,383$ & 2 & 0,115 \\
33 & Canteras & -1 & $-0,437$ & 4 & 0,091 \\
34 & Pto Berrío & -1 & $-0,426$ & 0 & 0,058 \\
35 & Peñas Blancas & -4 & $-0,253$ & -1 & 0,108 \\
36 & Pto Libre & -2 & $-0,364$ & 10 & 0,104 \\
37 & Pto Salgar & $-0,413$ & -8 & $-0,091$ \\
38 & Colorados & $-0,29$ & 10 & 0,084 \\
39 & Pto Araújo & $-0,227$ & 9 & 0,065 \\
40 & Maldonado & -359 & 11 & $-0,173$ \\
\hline
\end{tabular}

Las correlaciones temporales entre el índice NAO y los caudales evidencian muy poca influencia en la cuenca Alta y Media, sólo cinco de las 41 estaciones presentan correlación positiva para rezagos también positivos, es decir que el fenómeno NAO se presenta después de los cambios hidrológicos, esto verifica la poca explicación que da la variable NAO a las alteraciones o cambios en el caudal. En la Tabla 5 se presentan los mayores coeficientes de correlación cruzada y los rezagos en meses para los índices MEI y NAO, vs los caudales. 


\section{Resultados del análisis de tendencia}

El análisis de tendencias se realizó mediante el programa Makesens y los resultados se corroboraron a través de Excel y SPSS. En la Tabla 6 se presenta el resumen de los resultados del cálculo de tendencias. Dentro de cada cuadro se muestra el valor de la pendiente de la recta de tendencia. Los cuadros grises indican tendencia negativa, los blancos tendencia positiva.

En términos generales para todas las estaciones, las tendencias de disminución de caudal son más intensas durante los meses de octubre y noviembre. Del total de 40 estaciones a las que se les realizó la prueba, 12 presentan una pendiente negativa de la serie de tendencia para todos los meses del año. Este hecho evidencia la disminución de la disponibilidad hídrica y hace pensar en escasez del recurso a largo plazo.

La tabla 6 muestra las estaciones más afectadas y con mayor credibilidad, por tener periodos largos de registro y valores altos de significancia. Según la tabla, la estación que evidencia mayor pendiente negativa durante todos los meses del año es la de Puerto Salgar, que alcanza valores negativos de pendiente mayores a 20 durante los meses de octubre y noviembre.

Tabla 6. Estaciones que presentan la mayor secuencia de pendientes negativas

\begin{tabular}{|lccccccccccccc|}
\hline Estación & $\mathrm{E}$ & $\mathrm{F}$ & $\mathrm{M}$ & $\mathrm{A}$ & $\mathrm{M}$ & $\mathrm{J}$ & $\mathrm{J}$ & $\mathrm{A}$ & $\mathrm{S}$ & $\mathrm{O}$ & $\mathrm{N}$ & $\mathrm{D}$ & $\mathrm{P}$ \\
\hline Cascada & $-0,6$ & $-0,1$ & $-0,6$ & $-0,4$ & $-0,5$ & $-0,6$ & $-0,5$ & $-0,6$ & $-1,4$ & $-0,5$ & $-0,4$ & $-0,5$ & $-0,6$ \\
Sala Bco & $-0,6$ & $-0,1$ & $-0,7$ & $-0,3$ & 0,3 & $-0,7$ & $-0,8$ & $-0,6$ & $-1,4$ & $-0,8$ & $-0,5$ & $-0,4$ & $-0,4$ \\
Pericon & $-1,3$ & $-0,8$ & $-1,2$ & $-1,1$ & 0,0 & $-0,6$ & $-0,9$ & $-0,8$ & $-1,9$ & $-1,3$ & $-0,9$ & $-0,8$ & $-0,8$ \\
Pte Bals & $-1,1$ & $-0,2$ & $-1,1$ & $-1,0$ & 0,4 & 0,1 & $-1,1$ & $-0,5$ & $-2,0$ & $-1,5$ & $-1,4$ & $-0,9$ & $-0,7$ \\
Paicol & $-1,4$ & $-1,3$ & $-1,0$ & $-0,1$ & 0,6 & $-0,2$ & $-0,7$ & $-1,0$ & $-2,4$ & $-1,7$ & $-1,2$ & $-0,8$ & $-0,8$ \\
Pte Mula & $-0,3$ & $-0,2$ & $-0,1$ & $-0,3$ & $-0,1$ & $-0,1$ & $-0,4$ & $-0,2$ & $-0,2$ & $-0,3$ & $-0,4$ & $-0,3$ & $-0,3$ \\
Nariño & $-2,5$ & $-1,4$ & $-0,4$ & $-1,4$ & $-2,2$ & $-2,4$ & $-2,8$ & $-4,2$ & $-2,5$ & $-4,9$ & $-8,7$ & $-0,7$ & $-2,6$ \\
Arrancap & $-4,0$ & $-2,9$ & $-1,3$ & $-0,7$ & $-2,9$ & $-4,5$ & $-4,5$ & $-5,1$ & $-3,4$ & $-5,4$ & $-10,5$ & $-2,1$ & $-3,8$ \\
Corralito & $-0,1$ & $-0,1$ & 0,0 & $-0,1$ & $-0,1$ & $-0,1$ & 0,0 & 0,0 & 0,0 & $-0,1$ & $-0,1$ & $-0,1$ & 0,0 \\
Pto Ver & $-6,2$ & $-3,4$ & $-1,4$ & $-2,9-10,3$ & $-6,9$ & $-7,1$ & $-8,5$ & $-3,7$ & $-6,5$ & $-11,2$ & $-4,4$ & $-6,3$ \\
PtoSal & $-8,9$ & $-6,0$ & $-5,0$ & $-12,1-14,1-15,5-14,0-13,6-10,4-20,4$ & $-23,6$ & $-10,0-13,9$ \\
Colorad & $-0,4$ & $-0,5$ & $-0,1$ & $-0,1$ & $-0,9$ & $-0,6$ & $-0,6$ & $-0,4$ & $-0,2$ & $-0,7$ & $-1,2$ & $-0,4$ & $-0,5$ \\
\hline
\end{tabular}

En la tabla 7 se presenta el resumen de las estaciones que alternan periodos de pendientes negativas y positivas. Se destaca la estación Payandé que presenta tendencia positiva durante todos los meses del año.

Tabla 7. Estaciones que alternan tendencias positivas y negativas

\begin{tabular}{|c|c|c|c|c|c|c|c|c|c|c|c|c|c|}
\hline ta & E & $\mathrm{F}$ & M & A & M & J & $\mathrm{J}$ & A & $\mathrm{s}$ & 0 & $\mathrm{~N}$ & D & $\mathrm{P}$ \\
\hline & $-0,2$ & & $-0,1$ & $-0,2$ & 0,1 & & & 0,0 & -01 & $-0,1$ & $-0,2$ & $\frac{1,2}{, 2}$ & \\
\hline & $-0,9$ & & & & & & & & & $-23,9$ & 9,3 & 15,4 & \\
\hline & 0,5 & & 1,2 & & & 0,3 & 0,7 & 0,9 & & & $-1,9$ & 0,1 & \\
\hline & 0,1 & & 0,6 & 0,1 & $-0,3$ & 0,0 & $-0,1$ & 0,0 & $-0,1$ & 0,3 & 0,5 & $-0,1$ & 0,0 \\
\hline & 2,2 & 2,2 & 2,1 & $-0,5$ & 0,0 & $-1,5$ & $-0,4$ & 0,6 & 0,9 & $-0,6$ & $-2,9$ & 0,4 & 0,4 \\
\hline & $-1,1$ & & $-11,4$ & $-9,8$ & $-21,3$ & $-8,9$ & $-13,9$ & 9,1 & 6,5 & $-4,2$ & $-3,9$ & 0,5 & $-6,2$ \\
\hline & 0,2 & & & $-0,5$ & 0,0 & & 0,5 & 0,1 & $-0,1$ & 0,2 & 0,1 & 0,6 & 0,2 \\
\hline & $n 4,3$ & & ,9 & 1,5 & 0,9 & 0,1 & $-1,1$ &, 2 & $-1,4$ & 2,4 &, 7 & 2,0 & 0,5 \\
\hline & 0,1 & & 1 & 0,0 & $-0,1$ & & 0,1 & 0 & 0 & 0,1 &, 1 &, 0 & 0,0 \\
\hline & 0,5 & & 0,8 & 0,1 & $-0,3$ & 3 & 0,5 & 4 & 0,0 & $-0,9$ & 1,8 & 0,1 & $0, \mathrm{C}$ \\
\hline & 0,4 & & 0,5 & 0,1 & 0,1 & 0 , & $-0,1$ & 0 &, 3 & 0,1 &, 3 & .4 & 0,2 \\
\hline & 0,0 & & 0,4 & 0,1 & 0,7 & & 0 & ,4 & 0,0 & $-0,2$ & 0,3 & 0,1 & 0,3 \\
\hline & 0,5 & & 8 & 0,1 & $-0,3$ & 3 & 0,5 & ,4 &, 0 & $-0,9$ & 1,8 & 0,1 & 0,0 \\
\hline & 0,4 & & 0,5 & 0,1 & 0,1 & 0,0 & $-0,1$ & 0,0 & 0,3 & $-0,1$ & 0,3 & 0,4 & \\
\hline & 0,5 & & & 0,2 & 0,3 & & 0,5 & 0,6 & 0,4 & 0,4 & 0,2 & 0,7 & \\
\hline & & & & 0,2 & 0,0 & & & 0,0 & $-0,1$ & 0,0 & & 20 & \\
\hline & $-0,1$ & & 0,1 & 0,2 & 0,2 & & , 1 & 0,1 & 0,1 & 0, & & 0,0 & \\
\hline & 0 & & 0,0 & 0,1 & 0,0 & & 1 & 0 & 0,0 & 0 & 0 & 0,0 & \\
\hline & $-0,3$ & & 0,1 & 0,1 & 0,0 & 0 & $-0,4$ & $-0,5$ & $-0,3$ & $-0,4$ & 0 & $-0,3$ & -0 , \\
\hline & 0,1 & 0 , & 0,1 & 0,2 & 0,2 & 0,2 & 0,0 & 0,0 & 0,1 & 0,0 & $-0,1$ & 0,0 & 0 , \\
\hline & $-0,1$ & 0 , & 0,1 & 0,2 & $-0,4$ & $-0,2$ & $-0,1$ & & & $-0,4$ & $-0,9$ & 0,1 & -0 , \\
\hline & 1 , & 1, & 0,4 & 0,9 & 1,2 & 0 , & 0,1 & & & $-0,9$ & 0,3 & 1,4 & 0,4 \\
\hline & $-0,8$ & 1,3 & 2,5 & 0,4 & $-0,9$ & -1 & $-0,1$ & -2 & & $-1,8$ & $-2,4$ & 1,7 & \\
\hline & 31 & & 29,9 & & & & & & & & & 27,9 & \\
\hline & -0 & & 0,6 & 0,6 & $-1,9$ & -0 & $-0,8$ & -1 & & $-1,3$ & $-3,3$ & $-0,7$ & $-0,9$ \\
\hline & $-0,1$ & 0,5 & 0,9 & $-0,7$ & $-1,9$ & $-0,8$ & $-1,3$ & $-1,9$ & $-0,4$ & $-2,9$ & $-4,1$ & 1,1 & $-1,1$ \\
\hline Ialdonado & & & & & & $-16,9$ & 6,4 & 2,4 & & & $-4,4$ & 4,5 & 15,0 \\
\hline
\end{tabular}

Estaciones como Vichecito y La Esperanza muestran altas pendientes negativas para el mes de Julio $(-30,667$ y -21.250 respectivamente), sin embargo su nivel de significancia no alcanza el $90 \%$, estos valores deben mirarse con mucho cuidado, dado que son series muy cortas a partir de las cuales no se pueden obtener pronósticos muy seguros, sin embargo se dejaron con el fin de establecer comparaciones.

La estación que presenta valores más exagerados en la pendiente es Peñas Blancas para los meses de abril, -31.8 y mayo, -44.1 , para octubre con -50.2 y de -51.9 para noviembre. Esto se explica fácilmente debido a los vacios que presenta la serie, con un total de datos perdidos de 7 años intercalados entre 1977 y 2003. 
La estación Maldonado presenta valores decrecientes para julio de -16.9 y para octubre con -13.1. Las demás estaciones presentan pendientes menores a -10 .

\section{CONCLUSIONES}

De 40 estaciones en estudio, 26 presentan correlación negativa con el fenómeno ENSO (mientras el ENSO se intensifica, el caudal disminuye). Esto quiere decir que el índice MEI explica en buena parte la ocurrencia de disminuciones de caudal. Sin embargo, es necesario mirar con mucho cuidado estos resultados, teniendo en cuenta que solamente cinco de ellos poseen un nivel de confianza mayor al 90\%. Esto sugiere que para las estaciones de Puente Garcés que pertenece al Río Suaza, Puente Santander, Purificación y Angostura, que pertenecen a la corriente del Río Magdalena y Puente Mulas del Río Neiva, la disminución de caudal sí se encuentra influenciada en buena parte por el fenómeno del Niño, mientras que para las otras estaciones la disminución en el caudal puede explicarse por muchos otros factores, tales como el uso de la tierra, la erosión, el crecimiento de la población y el aprovechamiento del recurso hídrico para riego, acueductos, hidroeléctricas, consumo humano, etc. La conjunción de estos factores requiere esfuerzos de investigación más precisos para un manejo más adecuado de las amenazas derivadas del fenómeno.

Si se observan mes a mes, todas las estaciones muestran correlación negativa con el fenómeno ENSO al menos durante los tres primeros meses del año, durante los siguientes trimestres algunas estaciones presentan correlación positiva, al final un $78 \%$ de las estaciones muestran correlación negativa con el dicho índice.

A través de los valores mensuales también se pueden observar correlaciones del orden de 0.7 para las estaciones de Puente Santander, Payandé, Nariño y Arrancaplumas durante el primer trimestre del año, mientras que hacia mitad de año, para los meses de mayo y julio, la estación Payandé alcanza valores de correlación con el índice MEl cercanos a 0.8.

Con relación al índice $\mathrm{MEI}$, las correlaciones muestran valores entre -0.15 y -0.505 , para rezagos negativos en promedio de tres meses. Las series más largas y por lo tanto las más confiables, presentan mayores valores de correlación, lo cual evidencia la posibilidad de que el fenómeno tenga alguna influencia del $\mathrm{MEI}$ en las series de caudales.

Se aprecia una escasa correlación con el índice NAO, ya que sólo cinco de las 40 estaciones presentan algún grado de correlación positivo entre 0.5 y 0.7 para diferentes meses del año, pero de éstas, a excepción de la estación Payandé, las que presentaron mayor correlación corresponden a series de tiempo cortas, de unos 9 años, que no demuestran el real comportamiento de la zona. Si se tiene en cuenta la serie total anual, sin dividirla en series mensuales, las cinco estaciones mencionadas presentan correlación (positiva), para rezagos también positivos, es decir que el fenómeno NAO se presenta después de los cambios hidrológicos. Esto verifica la poca explicación que da la variable NAO a las alteraciones o cambios en el caudal.

La disponibilidad hídrica en la cuenca del Magdalena se considera relevante, debido a que en esta zona se concentra la mayoría de la población, hecho que la hace más vulnerable frente a la escasez del recurso. Se estima una alta disminución del caudal hacia el 2015, por esta razón es conveniente tomar medidas que mejoren la conservación del recurso hídrico.

En términos generales para todas las estaciones, las tendencias de disminución de caudal son más intensas durante los meses de octubre y noviembre. Del total de 40 estaciones a las que se les realizó la prueba, 12 presentan una pendiente negativa de la serie de tendencia para todos los meses del año. Este he- 
cho demuestra la disminución de la disponibilidad hídrica y hace pensar en escasez del recurso a largo plazo.

La estación que presenta mayor pendiente negativa durante todos los meses del año es la de Puerto Salgar, que alcanza valores negativos de pendiente mayores durante los meses de octubre y noviembre.

Estaciones tales como Vichecito, La Esperanza y Peñas Blancas muestran pendientes negativas muy altas, pero estos valores son poco confiables, dado que son series muy cortas a partir de las cuales no se pueden obtener pronósticos muy seguros, sin embargo se utilizaron para establecer comparaciones.

La estación Maldonado presenta valores decrecientes para julio de -16.9 y para octubre con -13.1. Las demás estaciones presentan pendientes menores a -10 .

Es recomendable relacionar el caudal con otras variables tales como precipitación, temperatura, velocidad del viento, etc, ya que estos factores también tienen una alta incidencia en la disponibilidad hídrica y permiten esclarecer con mayor precisión las relaciones existentes entre las diferentes variables.

Es necesario enfatizar sobre las políticas ambientales tendientes a la conservación del recurso hídrico, tales como protección de zonas que producen agua, reforestación, conservación del suelo a través de rotación de cultivos y zonas de pastoreo, tratamiento de aguas servidas y campañas de información y concientización.

El presente trabajo finalmente corrobora los estudios realizados por Poveda (2005) en el que se detecta la disminución de caudal en los ríos durante los últimos decenios explicada por aumento en la temperatura y la humedad sin variación significativa de la precipitación.

\section{REFERENCIAS}

[1] ALVEAR, J. 2005. Manual del Río Magdalena. Cormagdalena, $250 \mathrm{p}$.

[2] Centro regional de información sobre desastres América Latina y el Caribe, CRID, 2002. Los efectos físicos y las amenazas asociadas a variaciones climáticas en Colombia. Página web consultada el 26 de abril de 2006. En: http://www.crid.or.cr/digitalizacion/pdf/spa/doc15906/doc15906-3.pdf.

[3] Centro de estudios y Experimentación de Obras Públicas de España CEDEX, Ministerio de Obras Públicas. Programa para el Cál-culo hidrometeorológico de aportaciones y crecidas CHAC, 2005. En: hercu-les.cedex.es/Chac/ - 2k. Página web consultada el día 30 de diciembre de 2005.

[4] COLCIENCIAS, 1999. Plan Estratégico 1999-2004. Página web, 5 de febrero de 2006, en: http://www. colciencias.gov.co/programas/ma/pdfs/planest.pdf.

[5] Grupo intergubernamental de expertos sobre el cambio climático, GIECC. 1997. Impactos regionales del cambio climático, evaluación de la vulnerabilidad, Noviembre de 1997.

[6] Instituto Geográfico Agustín Codazzi, IGAC. 1996. Diccionario Geográfico de Colombia, Colombia: IGAC, 5 tomos.

[7] Instituto de hidrología, meteorología y estudios ambientales IDEAM. 2002. Efectos naturales y socioeconómicos del fenómeno de El Niño en Colombia. Bogotá, Marzo de 2002.

[8] Colombia en el ambiente global. Página web, 5 de abril de 2005 en: http://www.ideam.gov.co/publica/ medioamb/cap2.pdf. 
[9] 1998. Estudio Nacional del Agua, Balance Hídrico y relaciones oferta demanda en Colombia. Indicadores de sostenibilidad proyectados al año 2016. Julio de 1998.

[10] IPCC. Pánel Intergubernamental sobre el cambio climático, 1995. Conclusiones del Segundo Reporte de Evaluación del PICC, Página web, mayo 20 de 2006 en: //www.cce.org.mx/cespedes/publicaciones/cuadernos/c1_c1.html.

[11] NOAA, Programa de paleoclimatología. La sequía en América del Norte, una paleo perspectiva. 2003. Página web http://wdc.cricyt.edu.ar/paleo/es/drought/drght_home.html, consultada en abril de 2006 .

[12] POVEDA, J.G. 2005. El fenómeno del Niño/Oscilación del Sur y su influencia sobre el clima de Colombia. Febrero de 2005.

[13] POVEDA, G., MESA O. J. 1996. Las fases extremas del ENSO - El Niño y La Niña - y su influencia sobre la hidrología de Colombia. Revista de Ingeniería Hidráulica en México, 1996. Vol. XI, No. 1, 21-37.

[14] POVEDA G., MESA, O. J. y CARVAJAI L. F. 1997. Introducción al clima en Colombia. Universidad Nacional de Colombia, Facultad de Minas Sede Medellín. Medellín: Unal, 390 p.

[15] Prodiversitas. Programa Panamericano de Defensa y Desarrollo de la Diversidad biológica, cultural y social. 2005. El fenómeno ENOS, calentamiento global y cambio climático. Página web, marzo 20 de 2005 en: http://www.prodiversitas.bioetica.org/ des41.htm

[16] ROGERS, J.C. 1984. La asociación entre la oscilación de Atlántico Norte y la oscilación meridional en el hemisferio norteño. Citado por Poveda G., Mesa, O. J. y Carvajal L. F. 1997.
[17] SARMIENTO, R. 2004. Colombia: desarrollo excluyente sin sostenibilidad. Página web consultada el día 2 de abril de 2005, en: http://www.redvoltaire. net/article2212.html. 